\title{
Failure to Rescue and Disparities in Emergency General Surgery
}

David Metcalfe LLB MRCP MRCS ${ }^{1}$, Manuel Castillo-Angeles MD MPH2,5, Olubode A. Olufajo MD $\mathrm{MPH}^{3}$, Arturo J. Rios-Diaz MD ${ }^{4}$, Ali Salim MD2,5, Adil H. Haider MD ${ }^{2,5}$, Joaquim M. Havens MD ${ }^{2,5}$.

1. Nuffield Department of Orthopaedics, Rheumatology and Musculoskeletal Sciences (NDORMS), University of Oxford, John Radcliffe Hospital, Headley Way, Oxford, OX3 9BU, United Kingdom.

2. Center for Surgery and Public Health (CSPH), One Brigham Circle, Boston, MA 02115, USA.

3. Department of Surgery, Barnes Jewish Hospital, Washington University School of Medicine, St Louis, Missouri, USA.

4. Department of Surgery, Thomas Jefferson University Hospital, 909 Walnut St, Philadelphia, PA 19107, USA.

5. Division of Trauma, Burn, and Surgical Critical Care, Brigham \& Women's Hospital, 75 Francis Street, Boston, MA 02115, USA.

\section{Corresponding author}

Dr. David Metcalfe, LLB MRCP MRCS

Clinical Research Fellow in Musculoskeletal Trauma

Kadoorie Centre for Critical Care Research

University of Oxford, Oxford, OX3 9DU

United Kingdom

+447800850000

david.metcalfe@balliol.ox.ac.uk

\section{Author contributions}

DM designed the study, performed the initial data analyses, and drafted the manuscript. MCA updated the analysis. MCA, OAO, ARD, AS, AHH, and JMH contributed to the study design, data interpretation, and made critical revisions to the manuscript.

\section{Funding}

The lead author undertook this project as part of a Royal College of Surgeons of England Fulbright Scholarship.

\section{Key words}

Failure to rescue; disparities; emergency general surgery; acute care surgery. 
Background: Racial and socioeconomic disparities are well documented in emergency general surgery (EGS) and have been highlighted as a national priority for surgical research. The aim of this study was to identify whether disparities in the EGS setting are more likely to be caused by major adverse events (e.g. venous thromboembolism) or failure to respond appropriately to such events.

Methods: A retrospective cohort study was undertaken using administrative data. Emergency general surgery cases were defined using ICD-9-CM diagnostic codes recommended by the American Association for the Surgery of Trauma (AAST). The data source was the National Inpatient Sample 2012-13, which captured a 20\% stratified sample of discharges from all hospitals participating in the Healthcare Cost and Utilization Project. The outcomes were major adverse events (MAEs), in-hospital mortality, and failure to rescue (FTR).

Results: There were 1,345,199 individual patient records available within the NIS. There were 201,574 admissions (15.0\%) complicated by a MAE and 12,006 of these (6.0\%) resulted in death. The FTR rate was therefore 6.0\%. Uninsured patients had significantly higher odds of MAEs (adjusted odds ratio [aOR] 1.16, 95\% confidence interval [CI] 1.13-1.19), mortality (1.28, 1.16-1.41), and FTR $(1.20,1.06-1.36)$ than those with private insurance. Although Black patients had significantly higher odds of MAEs (aOR 1.14, 95\% CI 1.13-1.16), they had lower mortality $(0.95,0.90-0.99)$ and failure to rescue $(0.86,0.80-0.91)$ than White patients.

Conclusions: Uninsured EGS patients are at increased risk of MAEs but also the failure of healthcare providers to respond effectively when such events occur. This suggests that MAEs and FTR are both potential targets for mitigating socioeconomic disparities in the setting of EGS. 


\section{Introduction}

Racial and socioeconomic disparities are increasingly recognized as important public health issues in the United States(1). It has been estimated that racial disparities account for 83,000 excess deaths(2) and cost over $\$ 250$ billion(3) every year. Disparities have been demonstrated across a range of surgical settings and highlighted as a national priority for surgical research(4).

Emergency general surgery (EGS) encompasses a group of patients presenting with acute surgical conditions. This population is particularly important as they are at higher risk of medical error, complications, and death than elective surgical patients(5-8). The proportion of EGS patients that experience complications in hospital has been estimated at between 15 and $50 \%(7,9)$. A number of studies have found that EGS patients are more likely to be uninsured or from minority racial groups(10). It has also been reported that lack of insurance and Black race are independent predictors of death after an EGS diagnosis(11) (12).

Healthcare disparities are typically explored using administrative datasets and previous studies have been limited to using in-hospital mortality as their primary outcome(11, 13-16). However, it is unclear whether this outcome truly reflects differences in surgical care, as many in-hospital deaths are unavoidable(17). One potential solution is to use "failure to rescue" (FTR), which is an emerging quality metric that has been shown to be more sensitive to differences in healthcare quality(18). Failure to rescue occurs when a patient dies as a result of a major adverse event (MAE) that developed in hospital and so could reflect failure to promptly recognize and treat developing complications(19). For example, the survival of a patient with post-operative pneumonia might depend on regular measurement of vital signs, early nursing recognition of deterioration, assessment by a doctor with sufficient experience to reach the correct diagnosis, prompt administration of antibiotics, and careful monitoring for signs of subsequent deterioration. It is therefore possible that FTR could be one mechanism leading to disparities in the EGS setting.

The aim of this study was to identify whether disparities in the EGS setting are more likely to be caused by major adverse events (e.g. venous thromboembolism) or failure to respond appropriately to such events. We hypothesized that disparities in EGS outcomes can be partially explained by differences in FTR. 


\section{Methods}

A retrospective cohort study was undertaken using administrative data. The study was approved by the Partners Healthcare Institutional Review Board (reference 2015P001722).

\subsection{Data source}

The National Inpatient Sample (NIS) is maintained by the Healthcare Cost and Utillization Project (HCUP) and is the largest publicly accessible all-payer patient database in the US. Between 2012 and 2013, the NIS captured a 20\% stratified sample of discharges from all hospitals participating in HCUP. There are approximately 6 million inpatient episodes recorded within the NIS each year, which can be weighted to provide estimates for around 35 million hospital admissions.

\subsection{Case selection}

All cases within the NIS were extracted that had a primary diagnosis consistent with the EGS definition provided by the American Association for the Surgery of Trauma (AAST)(20). These diagnoses are presented in Appendix 1. Cases were identified using a previously published list of International Classification of Diseases, 9th Revision, Clinical Modification (ICD-9-CM) diagnostic codes(21). Patients transferred between institutions were excluded to avoid inpatient episodes being inappropriately duplicated within the dataset.

\subsection{Variables and outcomes}

The patient-level characteristics were age, sex, race, payment source, median household income, admission source, weekend admission, and disease severity. Charlson comorbidity index (CCI) has been shown to predict mortality in the EGS population(22) and was determined from ICD-9-CM codes using the ICDPIC module in Stata(23). Disease severity is a variable provided within the NIS and estimates mortality risk along a four-point scale using All Patient Refined Diagnosis Related Groups (APRDRGs). The hospital-level characteristics were geographic region, rural location, teaching status, and hospital bed size.

The outcomes were major adverse events (MAEs), mortality, and FTR. Major adverse events were identified from ICD-9-CM codes used for this purpose by other studies(21): cerebrovascular accident (CVA), pneumonia, pulmonary embolus (PE), acute respiratory distress syndrome (ARDS), renal failure, urinary tract infection (UTI), myocardial infarction (MI), sepsis, septic shock, and cardiac arrest. The full list of ICD-9-CM codes used to define MAEs are available in an earlier publication(24). There are a number of operational definitions of FTR used within the patient safety literature, which are fundamentally similar 
but are based in different patient groups and use different MAEs to define their denominator and numerator populations(25). In common with all earlier definitions, FTR was defined as the odds of in-hospital mortality following a MAE: [mortality amongst cases with coded MAEs]/[all cases with coded MAEs].

\subsection{Statistical analyses}

Categorical variables were compared using Chi-square tests and continuous variables with Wilcoxon rank sum tests. Outcomes were adjusted for patient- and hospital-level factors using multivariable logistic regression. The covariates were age, sex, race, payer status, CCI, weekend admission, median household income, and hospital bed size. Raw data were presented from the NIS rather than relying on discharge weights, which are intended for use to create nationally representative estimates. Statistical analyses were conducted using Stata 13.0 (College Station, TX, USA) with the threshold for statistical significance set at $\mathrm{p}<0.05$.

\section{Results}

There were 1,345,199 individual EGS records available in the NIS and he characteristics of this population are shown in Table 1 . The three most frequently encountered diagnoses were "other cellulitis and abscess" (ICD-9-CM 682.1-9, 9.4\%), "diverticula of small or large intestine with diverticulitis or hemorrhage" (562.1-3, 5.4\%), and "intestinal obstruction without hernias" $\left(560 .^{*}, 4.7 \%\right)$. Just under half (49.5\%) of these patients underwent an operation during their hospitalization. 201,574 admissions (15.0\%) were complicated by a MAE and $12,006(6.0 \%)$ of these resulted in death. The proportion of patients that were not successfully "rescued" was therefore 6.0\%.

\subsection{Insurance status}

Table 2 shows the association between insurance status and outcomes. In the unadjusted analyses, uninsured patients had the lowest proportion of MAEs (8.6\% versus privately insured $9.5 \%$ and publicly insured $19.2 \%, \mathrm{p}<0.001)$, mortality $(0.5 \%$ versus privately insured $0.6 \%$ and publicly insured $1.9 \%, \mathrm{p}<0.001)$, and FTR (3.8\% versus privately insured $4.2 \%$ and publicly insured 6.6\%, p<0.001). However, in the adjusted analyses, uninsured patients had significantly higher odds of MAEs (adjusted odds ratio 1.16, 95\% confidence interval 1.131.19), mortality $(1.28,1.16-1.41)$, and FTR $(1.20,1.06-1.36)$ than those with private insurance. 
Table 3 shows that a greater proportion of Black patients experienced MAEs (17.1\%) than White (15.4\%) and Hispanic (10.9\%) patients. However, both mortality (1.2\%) and FTR (4.9\%) were lower than amongst White patients (1.4\% and $6.2 \%$ respectively). In keeping with the unadjusted analyses, black patients had significantly higher odds of complications (adjusted odds ratio 1.14, 95\% confidence interval 1.13-1.16) but lower mortality $(0.95,0.90$ $0.99)$ and FTR $(0.86,0.80-0.91)$ than White patients.

Hispanic patients experienced the lowest proportion of MAEs (10.9\% versus White 15.4\% and Black 17.1\%) and mortality (0.8\% versus White $1.4 \%$ and Black $1.2 \%)$. In the adjusted analyses, they had lower odds of MAE (adjusted odds ratio 0.83, 95\% confidence interval 0.82-0.85) and mortality $(0.82,0.77-0.87)$ than both White and Black patients. However, the adjusted odds of FTR amongst Hispanic patients (0.94, 0.87-1.02) was not significantly different from that of White patients.

\section{Discussion}

A number of mechanisms have been proposed to explain surgical outcome disparities. Potential factors at the patient-level include differences in age, sex, health insurance, social support, and geographical barriers to accessing healthcare. At the provider-level, reimbursement concerns might limit the resources made available to uninsured patients(26, 27). Although implicit biases have been demonstrated amongst US surgeons, there is little evidence that these inform surgical decision-making(26). It has however been proposed that Black and uninsured patients are more likely to cluster in hospitals that are underresourced(13) and have worse outcomes(11). It is therefore possible that differences in clinical healthcare quality might contribute to racial and socioeconomic disparities. However, previous studies have not explored this excess mortality further by separately reporting differences in MAEs and FTR.

Our study found that lack of insurance is an independent risk factor for in-hospital mortality across the whole EGS population. The odds of MAE and FTR were also higher and so may contribute to excess mortality in this group. This study did not find evidence of racial disparities in EGS mortality and so could not invoke MAE or FTR as explanatory factors.

\subsection{Insurance status}

The finding that lack of insurance is an independent risk factor for mortality is consistent with other EGS studies(14, 21, 28, 29). A number of explanations have been proposed for worse outcomes amongst uninsured EGS patients. These include reduced access to elective 
surgery(30), delayed clinical presentation(31), barriers to accessing specialist centers(30), and differences in surgical interventions offered $(32,33)$. Our study suggests that both excess MAEs and FTR might contribute to the higher odds of mortality amongst uninsured EGS patients. This suggests that further efforts to mitigate disparities must focus both on reducing complications and enhancing the provider response when they occur.

\subsection{Race}

In this study, Black race was an independent risk factor for developing MAEs. A number of earlier studies have also suggested that MAEs might occur disproportionately amongst Black patients in the post-operative period. For example, there is evidence that they are more likely to be re-admitted to hospital within 30 days following discharge(8).

Although other studies have reported higher mortality amongst Black EGS patients, this was not evident in our analysis. It has recently been shown that racial disparities were evident in earlier years of the NIS (e.g. the 1993 EGS cohort) but appeared to have dissipated from 2003 onwards(28). In our study, Black patients had lower odds mortality than their White counterparts. Although this difference was statistically significant, the effect size was small (adjusted odds ratio 0.95, 95\% confidence interval 0.90-0.99). As studies with large sample sizes and multiple comparisons risk overstating statistical significance(34), this might suggest a marginal survival benefit for Black patients or simply equivalent odds of mortality as their White counterparts. This is particularly likely given that the adjusted odds of mortality were significantly higher for Black patients in the sub-group analysis using only the top three EGS diagnoses.

\subsection{Limitations}

The principal limitation of this study is that it relied upon an administrative database. Although the NIS is an established research tool and represents the largest sample of discharge records from the across the United States, it does not capture some important variables, e.g. physiological and biochemical data. This may have implications for the adequacy of risk adjustment for differences in patient case mix. Administrative sources are also subject to coding variability with previous studies showing that choice of dataset can dramatically influence study findings(35). Similarly, it cannot be determined using NIS data whether or not any given MAE was actually present on admission and so should be discounted as an EGS "complication". It will therefore be necessary to replicate this study in other datasets, such as individual State Inpatient Databases (some of which include a "present on admission" variable) and the American College of Surgeons National Surgical Quality 
Improvement Program (ACS NSQIP) database. However, these databases are not in themselves superior to the NIS and will carry their own limitations. For example, although ACS NSQIP includes more variables for risk adjustment, it only captures patients with a limited number of EGS diagnoses(36).

The other principle limitation is that EGS diagnoses were treated as a homogenous group of disorders, which masks considerable heterogeneity. For example, patients requiring emergency laparotomy might exhibit a different profile of MAEs (and possibly likelihood of "rescue" post-MAE) than those admitted with adhesional small bowel obstruction. Although disease severity appears as a variable in the NIS along a four-point scale, there is not yet a validated hierarchy of EGS disease severity on which to base this analysis(37). We addressed this limitation by presenting a sub-group analysis of the most frequently occurring EGS diagnoses. However, there is likely to be considerable residual diagnostic heterogeneity, even within the same ICD-9-CM code, e.g. "other cellulitis and abscess" could potentially include patients with simple cellulitis to Fournier's gangrene. It is also possible that research findings might vary based on the precise definition of FTR used(25) as this would impact substantially on the denominator and numerator populations.

\subsection{Conclusion}

This study found that both increased MAEs and FTR may contribute to excess mortality for uninsured EGS patients. Although this finding should be tested in other datasets, it suggests that both MAEs and FTR are potential targets for mitigating socioeconomic disparities.

\section{References}

1. Smedley B, Stith A, Nelson A Unequal Treatment: Confronting Racial and Ethnic Disparities in Healthcare. Washington DC: 2003.

2. Satcher D, Fryer GE, Jr., McCann J, Troutman A, Woolf SH, et al. What if we were equal? A comparison of the black-white mortality gap in 1960 and 2000. Health Aff (Millwood) 2005:24:459-464.

3. LaVeist TA, Gaskin D, Richard P Estimating the economic burden of racial health inequalities in the United States. Int J Health Serv 2011:41:231-238.

4. Haider AH, Dankwa-Mullan I, Maragh-Bass AC, Torain M, Zogg CK, et al. Setting a National Agenda for Surgical Disparities Research: Recommendations From the National Institutes of Health and American College of Surgeons Summit. JAMA Surg 2016.

5. Gawande AA, Studdert DM, Orav EJ, Brennan TA, Zinner MJ Risk factors for retained instruments and sponges after surgery. N Engl J Med 2003:348:229-235.

6. Havens JM, Peetz AB, Do WS, Cooper Z, Kelly E, et al. The excess morbidity and mortality of emergency general surgery. J Trauma Acute Care Surg 2015:78:306-311. 
7. Kwan TL, Lai F, Lam CM, Yuen WC, Wai A, et al. Population-based information on emergency colorectal surgery and evaluation on effect of operative volume on mortality. World J Surg 2008:32:2077-2082.

8. Havens JM, Olufajo OA, Cooper ZR, Haider AH, Shah AA, et al. Defining Rates and Risk Factors for Readmissions Following Emergency General Surgery. JAMA Surg 2016:151:330336.

9. Scott JW, Olufajo OA, Brat GA, Rose JA, Zogg CK, et al. Use of National Burden to Define Operative Emergency General Surgery. JAMA Surg 2016:e160480.

10. Tsai TC, Orav EJ, Joynt KE Disparities in surgical 30-day readmission rates for Medicare beneficiaries by race and site of care. Ann Surg 2014:259:1086-1090.

11. Hall EC, Hashmi ZG, Zafar SN, Zogg CK, Cornwell EE, 3rd, et al. Racial/ethnic disparities in emergency general surgery: explained by hospital-level characteristics? Am J Surg 2015:209:604-609.

12. Shahan CP, Bell T, Paulus E, Zarzaur BL Emergency general surgery outcomes at safety net hospitals. J Surg Res 2015:196:113-117.

13. Hoehn RS, Wima K, Vestal MA, Weilage DJ, Hanseman DJ, et al. Effect of Hospital SafetyNet Burden on Cost and Outcomes After Surgery. JAMA Surg 2016:151:120-128.

14. Schwartz DA, Hui X, Schneider EB, Ali MT, Canner JK, et al. Worse outcomes among uninsured general surgery patients: does the need for an emergency operation explain these disparities? Surgery 2014:156:345-351.

15. Silber JH, Williams SV, Krakauer H, Schwartz JS Hospital and patient characteristics associated with death after surgery. A study of adverse occurrence and failure to rescue. Med Care 1992:30:615-629.

16. Zogg CK, Jiang W, Chaudhary MA, Scott JW, Shah AA, et al. Racial disparities in emergency general surgery: Do differences in outcomes persist among universally insured military patients? J Trauma Acute Care Surg 2016.

17. Hogan H, Zipfel R, Neuburger J, Hutchings A, Darzi A, et al. Avoidability of hospital deaths and association with hospital-wide mortality ratios: retrospective case record review and regression analysis. BMJ 2015:351:h3239.

18. Taenzer AH, Pyke JB, McGrath SP A review of current and emerging approaches to address failure-to-rescue. Anesthesiology 2011:115:421-431.

19. Johnston MJ, Arora S, King D, Bouras G, Almoudaris AM, et al. A systematic review to identify the factors that affect failure to rescue and escalation of care in surgery. Surgery 2015:157:752-763.

20. Shafi S, Aboutanos MB, Agarwal S, Jr., Brown CV, Crandall M, et al. Emergency general surgery: definition and estimated burden of disease. J Trauma Acute Care Surg 2013:74:10921097.

21. Shah AA, Haider AH, Zogg CK, Schwartz DA, Haut ER, et al. National estimates of predictors of outcomes for emergency general surgery. J Trauma Acute Care Surg 2015:78:482-490; discussion 490-481.

22. St-Louis E, Iqbal S, Feldman LS, Sudarshan M, Deckelbaum DL, et al. Using the ageadjusted Charlson comorbidity index to predict outcomes in emergency general surgery. J Trauma Acute Care Surg 2015:78:318-323.

23. Clark DE, Osler TM, Hahn DR ICDPIC: Stata module to provide methods for translating International Classification of Diseases (Ninth Revision) diagnosis codes into standard injury categories and/or scores. Boston College, 140 Commonwealth Avenue, Chestnut Hill, MA 02467, USA: 2010.

24. Metcalfe D, Castillo-Angeles M, Rios Diaz A, Havens JM, Haider A, et al. Is there a "weekend effect" in emergency general surgery? Journal of Surgical Research 2017:In press. 25. Silber JH, Romano PS, Rosen AK, Wang Y, Even-Shoshan O, et al. Failure-to-rescue: comparing definitions to measure quality of care. Med Care 2007:45:918-925. 
26. Haider AH, Schneider EB, Sriram N, Dossick DS, Scott VK, et al. Unconscious race and social class bias among acute care surgical clinicians and clinical treatment decisions. JAMA Surg 2015:150:457-464.

27. Moser JW, Applegate KE Imaging and insurance: do the uninsured get less imaging in emergency departments? J Am Coll Radiol 2012:9:50-57.

28. Armenia SJ, Pentakota SR, Merchant AM Socioeconomic factors and mortality in emergency general surgery: trends over a 20-year period. Journal of Surgical Research 2017:212:178-186.

29. Zogg CK, Scott JW, Jiang W, Wolf LL, Haider AH Differential access to care: The role of age, insurance, and income on race/ethnicity-related disparities in adult perforated appendix admission rates. Surgery 2016:160:1145-1154.

30. Stone ML, LaPar DJ, Mulloy DP, Rasmussen SK, Kane BJ, et al. Primary payer status is significantly associated with postoperative mortality, morbidity, and hospital resource utilization in pediatric surgical patients within the United States. J Pediatr Surg 2013:48:8187.

31. Scott JW, Rose JA, Tsai TC, Zogg CK, Shrime MG, et al. Impact of ACA Insurance Coverage Expansion on Perforated Appendix Rates Among Young Adults. Med Care 2016:54:818-826.

32. Vogel TR, Cantor JC, Dombrovskiy VY, Haser PB, Graham AM AAA repair: sociodemographic disparities in management and outcomes. Vasc Endovascular Surg 2008:42:555-560.

33. Zak Y, Rhoads KF, Visser BC Predictors of surgical intervention for hepatocellular carcinoma: race, socioeconomic status, and hospital type. Arch Surg 2011:146:778-784.

34. Perry DC, Parsons N, Costa ML 'Big data' reporting guidelines: how to answer big questions, yet avoid big problems. Bone Joint J 2014:96-B:1575-1577.

35. Schlussel AT, Delaney CP, Maykel JA, Lustik MB, Nishtala M, et al. A National Database Analysis Comparing the Nationwide Inpatient Sample and American College of Surgeons National Surgical Quality Improvement Program in Laparoscopic vs Open Colectomies: Inherent Variance May Impact Outcomes. Dis Colon Rectum 2016:59:843-854.

36. Nandan AR, Bohnen JD, Sangji NF, Peponis T, Han K, et al. The Emergency Surgery Score (ESS) Accurately Predicts the Occurrence of Postoperative Complications in Emergency Surgery Patients. J Trauma Acute Care Surg 2017.

37. Shafi S, Aboutanos M, Brown CV, Ciesla D, Cohen MJ, et al. Measuring anatomic severity of disease in emergency general surgery. J Trauma Acute Care Surg 2014:76:884-887. 